\title{
25I seeds implantation for lymph nodes metastasisfrom radioactive iodine-refractory differentiated thyroid carcinoma: A short-term efficacy and dosimetry study
}

Guoxu Zhang ( $\nabla$ zhangguoxu502@sina.com )

General hospital of northern theater command

Wenwen Zhang

General hospital of northern theater command

Shanshan Hao

General hospital of northern theater command

\section{Zhiguo Wang}

General hospital of northern theater command

Liqiu Ji

General hospital of northern theater command

\section{Xiangyan Ge}

General hospital of northern theater command

Gen Li

General hospital of northern theater command

\section{Youchao Wang}

General hospital of northern theater command

\section{Research Article}

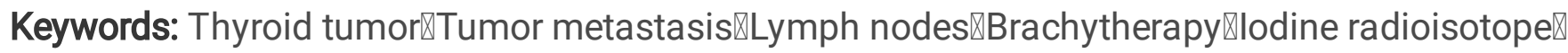

Radiation dose

Posted Date: February 1st, 2022

DOI: https://doi.org/10.21203/rs.3.rs-1310697/v1

License: (1) This work is licensed under a Creative Commons Attribution 4.0 International License.

Read Full License 


\section{Abstract}

Purpose: To evaluate the efficacy and safety of $125 \mathrm{I}$ seeds implantation for lymph nodes metastasis (LNM) from radioactive iodine-refractory differentiated thyroid carcinoma (RAIR-DTC), and to verify the computer three-dimensional treatment planning system (TPS) from the dosimetry accuracy in assisting seeds implantation to treat LNM.

Methods and materials: Retrospective analysis was performed on 17 RAIR-DTC patients with LNM admitted to the General Hospital of Northern Theater Command from December 2016 to January 2019 (8 males, 9 females, median age 58 years). All patients underwent preoperative TPS planning design, CTguided puncture and $125 \mathrm{I}$ seeds implantation (seed activity 14.8-25.9 MBq). The dosimetric results of postoperative validation were compared with those of preoperative planning, including the dosimetric parameters such as target volume before and after surgery and the dose received by $90 \%$ and $100 \%$ gross tumor volume (GTV) (D90, D100),the percentage of GTV receiving $100 \%$ and $150 \%$ prescription dose (V100, V150), homogeneity index (HI). All patients underwent CT after 6 months to compare the LNM size, serum thyroglobulin $(\mathrm{Tg})$ level, and the improvement of complications before and after treatment. Efficacies were divided into complete remission (CR), partial remission (PR), stable disease (SD), progressive disease (PD). Paired $t$ test or Wilcoxon signed rank test were used to analyze the data.

Results: Among 17 patients, a total of 226125 I radioactive seeds were implanted. Among them, 1 achieved CR, 10 achieved PR, 4 were with SD, 2 were with PD. The median diameter of LNM was $1.40(0.65,3.05) \mathrm{cm}$ before treatment and $0.40(0.21 \otimes 0.91) \mathrm{cm} 6$ months after treatment $(z=-3.95, P<0.05)$. The median $\mathrm{Tg}$ before treatment was $23.50(20.94,72.92) \mu \mathrm{g} / \mathrm{L}$ and 8.90(3.20,40.22) $\mu \mathrm{g} / \mathrm{L} 6$ months after treatment $(z=-5.009, P<0.001) ; \mathrm{Tg}$ antibody were all negative. There were $90.90 \%(20 / 22)$ of patients had slightly lower D90 than the rescription dose ((12 378.8 \pm 3 182.0) vs (12 497.8 \pm 1686.4$) c G y ; t=0.251$, $\mathrm{P}>0.05)$. The postoperative dose parameters D100 and V150 ((6 881.5 \pm 1381.8$) \mathrm{cGy},(58.5 \pm 18.40) \%)$ were both lower than that of preoperative plan ((8 085.8 \pm 2 330.0) cGy, $(66.5 \pm 17.70) \%$; $t$ values: 8.913, 3.032 , both $P<0.05)$, and there was no significant differences in the number of implanted particles, PTV, $\mathrm{V} 100$ and $\mathrm{HI}(\mathrm{t} / \mathrm{Z}=-0.593,-1.604,1.493,-0.663$, respectively, all $\mathrm{P}>0.05)$.

Conclusion: According to the TPS preoperative plan, $125 \mathrm{I}$ seeds implantation for treating RAIR-DTC LNM can achieve the expected dose distribution, and the short-term tumor local control is effective. It is a safe and effective treatment method.

\section{Introduction}

Thyroid carcinoma is one of the most common malignant tumors in the endocrine system, and differentiated thyroid carcinoma (DTC) accounts for about 90\% [1]. Most DTC surgeries supplemented with postoperative thyroid stimulating hormone (TSH) suppression or radioactive iodine (RAI) treatment have a good prognosis, but some tumor cells lose iodine capacity or decrease the expression of TSH receptors, forming radioactive iodine-refractory differentiated thyroid carcinoma (RAI-refractory DTC, 
RAIR-DTC), the 10-year survival rate of this part of patients is less than $10 \%$ [2]. Finding an effective treatment for RAIR-DTC has always been a hot topic in the field of thyroid cancer. At present, the commonly used methods for the treatment of RAIR-DTC include targeted drug therapy, local reoperation, chemotherapy, external radiation therapy, immunotherapy, gene therapy, etc. Part of lymph node metastases are often due to hidden locations or limitations of important anatomical structures (such as main trachea, large blood vessels, etc.), causing patients to lose the opportunity for surgery. In recent years, radioactive seed implantation has been widely used in the local treatment of malignant tumors. Considering that dose distribution is the most direct and important factor affecting the efficacy of seed implantation [3], A reasonable pre-implantation plan can be carried out according to the treatment planning system (TPS) to achieve the expected dose distribution. This study explored the short-term efficacy and adverse effects of $125 \mathrm{I}$ seed implantation in the treatment of RAIR-DTC withlymph node metastases, and we compared the preoperative and postoperative dosimetry results of the computerized three-dimensional TPS-assisted seed implantation therapy, and evaluated the accuracy of the technology in guiding the dose distribution of seed implantation therapy.

\section{Materials And Methods Research object}

A total of 17 RAIR-DTC patients with lymph node metastasis who were hospitalized at the Northern Theater Command General Hospital and received 125I seed implantation from December 2016 to January 2019 were collected, including 8 males and 9 females, with a median age of 58 years. The range was 22 to 70 years old囚andall of themwere digonisedaspapillary thyroid carcinoma (PTC). 22 metastatic lymph nodes were diagnosed pathologically by needle biopsy, of which 5 patients had 2 lymph node metastases, 12 cases of them has 1 lymph node metastasis. Each patient took 131I with a cumulative dose of $>22.2 \mathrm{GBq}$ and received $125 \mathrm{I}$ seed implantation at least half a year after $131 \mathrm{I}$ treatment. (This study was reviewed and approved by the Medical Ethics Committee of our hospital, and all patients signed an informed consent form.)Inclusion criteria: (1) Complete thyroidectomy or near-total resection before operation, according to functional neck dissection and extended radical resection of cervical lymph nodes, confirmed by pathology as DTC metastasis, it was identified as RAIR-DTC according to the 2015 American Thyroid Association (ATA) guidelines [4]; (2) WBC count $\geq 3.5 \times 109 / \mathrm{L}, \mathrm{Hb} \geq 90 \mathrm{~g} / \mathrm{L}$, prothrombin activity $>40 \%$, PLT $\otimes 100 \times 109 / L$, normal electrocardiogram, serum alanine aminotransferase and serum aspartate aminotransferase in liver function test Enzyme value does not exceed 3 times the normal upper limit; (3) Karnofsky performance status (KPS) scor $\geq 70$, with detailed clinicopathological data and follow-up data, survival time of more than 1 year; (4) no further transfer. Exclusion criteria: (1) Thyroglobulin antibody (TgAb) positive; (2) Severe heart, lung, liver and kidney dysfunction; (3) Severe coagulation dysfunction; (4) General condition is very poor or cachexia. 


\section{Instrument}

A 64-slice CT with Discovery VCT PET/CT produced by GE in the United States was used for routine scanning. The implant gun and the push thimble are flat-head general-purpose types produced by Zhejiang Xiangshan Jinheng Machinery Manufacturing Co., Ltd. The 15-20 cm×18 G puncture needle produced by Yako Co.; Ltd. is used. The particle TPS is provided by Beijing Feitian Zhaoye Technology Co., Ltd. The $125 \mathrm{I}$ radioactive particle source is produced by Beijing Atom Technology Co., Ltd. The particle length is $4.5 \mathrm{~mm}$, the diameter is $0.8 \mathrm{~mm}$, and the fully enclosed titanium shell. The particle activity is $14.8-25.9 \mathrm{MBq}$, the half-life is $59.6 \mathrm{~d}$, and the tissue penetration distance is $17 \mathrm{~mm}$. Steam sterilization. The whole body $131 \mathrm{l}$ imaging adopts the Symbia T16 SPECT/CT instrument of Siemens company in Germany. Five items of thyroid function (free triiodothyronine, free thyroxine, TSH, TgAb, antithyroid peroxidase autoantibody) and thyroglobulin ( $\mathrm{Tg}$ ) are detected by chemiluminescence method. The instrument and kit are composed of Soling Diagnostics (Italy) Co., Ltd. provides: Soling-LIAISON Chemiluminescence Analyzer, Soling-LIAISON Kit.

\section{Preparation before surgery}

Complete various laboratory tests before surgery, including blood routine, $\mathrm{ABO}$ and $\mathrm{RH}$ blood types, coagulation, liver and kidney function, four items of infectious diseases, five items of thyroid function, $\mathrm{Tg}$, electrocardiogram, etc.; inform patients and their families complications and precautions that may occur during and after the operation and sign the informed consent; Stopdrinking for 2 4 hours before local anesthesia, establishing thevenous access; performing breathing training to reduce the intervention caused by the patient's respiratory activity during the operation Implant deviation; patients with pain intolerance can be given tramadol analgesia (oral 50-100 mg/time) $30 \mathrm{~min}$ before surgery, and codeine phosphate tablets (oral, $15-30 \mathrm{mg} /$ time) can be given for irritation cough.

\section{TPS plan}

Import the enhanced or plain CT scan images 1 week before the operation into the TPS system for design, avoiding important tissues and organs (such as large blood vessels, heart, spinal cord, etc.) as much as possible. Delineate the gross tumor volume (GTV) and planning target volume (PTV). The PTV range includes the tumor itself and surrounding tissues that may infiltrate (such as burr signs around the tumor). Set an appropriate prescription dose of 110 140 Gy and particle activity (14.8 25.9 MBq) [5], follow the principle of $0.5 \sim 1.0 \mathrm{~cm}$ between particles, $1 \mathrm{~cm}$ between needle tracks, Making $90 \%$ of GTV received prescription dose (D90) > matched peripheral dose (matched peripheral dose, MPD), and then calculate the number of particles and particle source distribution to simulate the best way to enter the needle. Obtain the preoperative planning parameters D90, D100, the volume percentage (V100, V150) of $100 \%$ and $150 \%$ of the prescribed dose received by GTV, and the dose-volume histogram (DVH). 


\section{Intraoperative operations}

Select different positions according to the lesion location, place an external positioning grating on the skin area corresponding to the lesion, select the puncture point by CT scan, and intramuscularly inject $2 \%$ lidocaine for local infiltration anesthesia, determine the depth and angle of the puncture path, and follow the TPS plan Needle cloth layer by layer. During the operation, the position and direction of the implanted needle were adjusted according to the CT scan, so that the depth of the needle into the lesion was through the center of the tumor and $0.5 \mathrm{~cm}$ from the edge of the tumor. Then, one particle was implanted every 0.5 to $1.0 \mathrm{~cm}$ in the backward way, and the particles were supplemented from the periphery to the center, and the needle was placed again referring to the position of the implanted needle, and the intraoperative real-time optimization until the implantation was completed. Care should be taken to avoid important blood vessels and nerves during implantation. During the operation, the patient's heart rate, blood pressure, respiration and blood oxygen saturation were monitored. At the same time, the patient's consciousness, pain, breathing, cough, hemoptysis and other conditions were observed, and timely symptomatic treatment was performed. After the operation is completed, press the puncture point for 1020 minutes.

\section{Post-operative verification plan}

After the operation is completed, the CT images are immediately transferred to TPS for postoperative verification and particle identification, and D90, D100, V100, V150, DVH, and homogeneity index (HI) are counted. $\mathrm{HI}=(\mathrm{VT}$, ref-VT, 1.5ref)/VT, ref, $\mathrm{VT}$, ref, $\mathrm{VT}$, 1.5ref is the volume of the target area receiving the prescribed dose, and the volume of the target area receiving $150 \%$ of the prescribed dose $(\mathrm{cm} 3)$; Larger, indicating that the target dose distribution is more uniform [6].

\section{Postoperative review and short-term efficacy evaluation}

(1) Efficacy judgment. Neck color Doppler ultrasound and neck CT were reviewed before treatment and 6 months after treatment to evaluate the effect of $125 \mathrm{I}$ seed implantation. According to the solid tumor efficacy evaluation standard (Response Evaluation Criteria in Solid Tumors, RECIST) 1.1 for efficacy evaluation [7]. هComplete remission (CR): The lesion completely disappeared, and there is no imaging suggestion of recurrence, tumor residual and metastasis, or only $125 \mathrm{I}$ particle metallic shadow; $₫$ Partial remission (PR): The sum of the short diameter of the target lesion is at least The baseline short diameter of the lesion (the sum of the smallest diameters measured before treatment) was reduced by $30 \%$; $\llbracket$ stable disease (SD): those who did not meet the criteria for either PR or progressive disease (PD); $\triangle P D$ : Based on the minimum sum of the short diameters of the target lesions during the entire study, the diameter sum increased by $\geq 20 \%$; or the absolute value of the diameter sum increased by $\geq 5 \mathrm{~mm}$ (the appearance of one or more new lesions is also regarded as PD).(2) Adverse reactions. After the operation, closely observe whether the patient has adverse reactions such as bleeding, hematoma, particle displacement, 
needle implantation, etc., and refer to the United States Tumor Radiotherapy Cooperation Group/European Organization for Research and Treatment of Cancer (Radiation Therapy Oncology Group/European Organization for Research and Treatment of Cancer). (RTOG/EORTC) Acute Radiation Response Scoring Standard evaluates skin radiation damage caused by radioactive seed implantation [8], observes and evaluates complications during operation, postoperative and follow-up.

\section{Statistical analysis}

Use IBM SPSS 19.0 software to process data. Quantitative data conforming to the normal distribution are expressed as $\pm \mathrm{s}$, quantitative data not conforming to the normal distribution are expressed as $\mathrm{M}$ ( $\mathrm{P} 25$, P75); qualitative data are expressed as frequency or percentage. The quantitative data before and after treatment were compared by paired $t$ test or Wilcoxon signed rank test. $P<0.05$ indicates that the difference is statistically significant.

\section{Results}

A total of 22 lesions in 17 patients were successfully performed seed implantation, with a single particle activity of 14.8-25.9 MBq and a total of 226 implants. The number of seeds implanted in the 20 lesions was consistent with the distribution of the TPS plan, and the coincidence rate was $90.90 \%$ (20/22). 2 lesions were not implanted as planned due to the patient's body movement and the closeness of the tumor to the anatomical structure of the large blood vessels in the neck.

After 6 months of follow-up examination, 1 case was CR, 10 cases were PR, 4 cases were SD, and 2 cases were PD. The median diameter of lymph node metastases before treatment was $1.4(0.6810 .60 .65,3.05)$ $\mathrm{cm}$, and after 6 months of treatment, it was $0.4(0.2 \varangle 4.70 .21,0.91) \mathrm{cm}(\mathrm{z}=-3.95, \mathrm{P}<0.05)$; treatment The median $\mathrm{Tg}$ in the last 6 months was $8.90(3.20,40.22) \mu \mathrm{g} / \mathrm{L}$, which was significantly lower than the median $\operatorname{Tg}[23.50(20.94,72.92) \mu \mathrm{g} / \mathrm{L}]$ before treatment $(\mathrm{z}=-5.009, \mathrm{P}<0.001) ; \mathrm{TgAb}$ were all Negative. After treatment, 3 patients with hoarseness and 5 patients with local tenderness were significantly relieved.

According to the TPS design, the $125 \mathrm{I}$ seed implantation basically achieved the expected dose distribution (Table 1). $90.90 \%$ (20/22) of the patients had D90 slightly lower than the prescribed dose, but the difference was not statistically significant $(t=0.251, P>0.05)$. In terms of dosimetry indicators, D100 and V150 were lower than the preoperative plan ( $t$ value: 8.913, 3.032, both $P<0.05$ ); the number of implanted particles, PTV, V100, HI were not statistically significant $(\mathrm{t} / \mathrm{Z}=-0.593,-1.64,1.493,-0.663$, all $P>0.05)$. Figure 1 shows a typical patient image.

\section{Table 1}

Dose validation results before and after 22 lesion particle implantation procedures in 17 patients with iodine-refractory differentiated thyroid cancer 


\begin{tabular}{|c|c|c|c|c|}
\hline Time & $\begin{array}{l}\text { Pre- } \\
\text { operative } \\
\text { planning }\end{array}$ & $\begin{array}{l}\text { Post-operative } \\
\text { verification }\end{array}$ & $T / Z$ & $P$ \\
\hline $\begin{array}{l}\text { Number of implanted } \\
\text { particles[grain;M(P25,P75)] }\end{array}$ & $\begin{array}{l}15.5 \\
\varangle 2.0 \sim 32.0 \rrbracket\end{array}$ & 14.5ه2.0 30.0区 & $\begin{array}{l}Z \\
=-0.593\end{array}$ & $P=0.553>0.05$ \\
\hline $\mathrm{PTV}\left[\mathrm{cm}^{3} \llbracket \mathrm{M}(\mathrm{P} 25, \mathrm{P} 75)\right]$ & $\begin{array}{l}1.4 \\
₫ 0.6 \sim 9.8 \rrbracket\end{array}$ & $1.4 \llbracket 0.6 \sim 10.6 \rrbracket$ & $\begin{array}{l}Z \\
=-1.604\end{array}$ & $P=0.109>0.05$ \\
\hline \multirow[t]{2}{*}{ D90冈cGy;x $\pm s \rrbracket$} & 12 & 12 & \multirow{2}{*}{$\begin{array}{l}\mathrm{t} \\
=0.251\end{array}$} & \multirow[t]{2}{*}{$P=0.402>0.05$} \\
\hline & $\begin{array}{l}497.8 \pm 1 \\
686.4\end{array}$ & $378.8 \pm 3182.0$ & & \\
\hline \multirow[t]{2}{*}{ D100』cGy;x $\pm s \rrbracket$} & 8 & 6 & \multirow{2}{*}{$\begin{array}{l}\mathrm{t} \\
=8.913\end{array}$} & \multirow[t]{2}{*}{$P=0.013<0.05$} \\
\hline & $\begin{array}{l}085.8 \pm 2 \\
330.0\end{array}$ & $881.5 \pm 1381.8$ & & \\
\hline V150冈\%;x $\pm s \rrbracket$ & $66.5 \pm 17.70$ & $58.5 \pm 18.40$ & $\begin{array}{l}\mathrm{t} \\
=3.032\end{array}$ & $P=0.035<0.05$ \\
\hline $\mathrm{V} 100 \rrbracket \% ; \mathrm{x} \pm \mathrm{s} \rrbracket$ & $94.5 \pm 8.27$ & $92.2 \pm 14.3$ & $\begin{array}{l}\mathrm{t} \\
=1.493\end{array}$ & $P=0.161>0.05$ \\
\hline $\mathrm{HI}[\mathrm{M}(\mathrm{P} 25, \mathrm{P} 75)]$ & $\begin{array}{l}0.3 \\
\rrbracket 0.2 \sim 0.4 \rrbracket\end{array}$ & $0.4 \rrbracket 0.3 \sim 0.5 \rrbracket$ & $\begin{array}{l}Z \\
=-0.663\end{array}$ & $P=0.508>0.05$ \\
\hline $\begin{array}{l}\text { Table1: D90 and D100 are th } \\
\text { (GTV), HI is the uniformity in } \\
\text { percentages of the GTV rece }\end{array}$ & $\begin{array}{l}\text { d doses rece } \\
\text { the planned } \\
\text { and } 150 \% \text { o }\end{array}$ & $\begin{array}{l}\text { ed by } 90 \% \text { and } 10 \\
\text { rget volume, and } \\
\text { ne prescribed dos }\end{array}$ & $\begin{array}{l}f \text { the gros } \\
\text { and V1 } \\
\text { pectively. }\end{array}$ & $\begin{array}{l}\text { tumor volume } \\
\text { are the }\end{array}$ \\
\hline
\end{tabular}

No serious bleeding or vascular embolism occurred during the operation. One case had a small amount of bleeding at the surgical site during the operation, which was relieved after intravenous injection of hemostatic drugs and compression to stop the bleeding; 2 cases had a skin reaction grade II reaction, which was improved after anti-inflammatory and external application of drugs, and no radioactive particles migrated to other tissues or organs Case.

\section{Discussion}

Cervical lymph node metastasis is the most common after PTC. Lymph node metastases are mostly located near the large blood vessels in the neck, around the trachea, and near the important nerves. The rapid growth of the tumor will cause invasion of the surrounding adjacent tissues. Patients often experience facial edema, pain, and voice. Clinical symptoms such as hoarseness and numbness. In recent years, the advantages of 125 I particle therapy have been gradually explored clinically. $125 \mathrm{I}$ continuously attenuates between tissues and releases $27.4 \rrbracket 31.4 \mathrm{keV} X$-rays and $35.5 \mathrm{keV} \mathrm{Y}$-rays [9], among which $\mathrm{Y}$-rays directly ionize tumor DNA single-strand or double-strand breaks, and X-rays generate oxygen free from indirect ionization. Base inactivation of tumor cells [10-11]. In addition, 125I particles act on some cancer cells in the quiescent phase (G0) phase, making them transformed into proliferation 
phase (G2 and M phase) cells that are easily killed after entering the division phase [10-11]. Recently, Chen Wei et al. [12] performed 125I particle therapy on 15 patients with neck recurrence after DTC. The volume reduction rate was $(83.5 \pm 16.9) \%$ after 12 months, and the postoperative serum Tg was 4.9 (0.7, 13.2) $\mu \mathrm{g} / \mathrm{L}$, which is significantly lower than preoperative [57.0 $(8.6,114.8) \mu \mathrm{g} / \mathrm{L}], 22$ high-enhancement lesions turned into low- or no-enhancement lesions, suggesting that the implantation of $125 \mathrm{I}$ particles in neck lesions has a large scope of application and strong operability , Good tolerance. In this study, 17 patients received $125 \mathrm{I}$ seed implantation, including $1 \mathrm{CR}, 10 \mathrm{PR}$, and $4 \mathrm{SD}$. After treatment, the size and $\mathrm{Tg}$ of the lesion were significantly lower than those before surgery, which were similar to the results of previous studies [13]. After treatment, the symptoms of 3 cases of hoarseness and 5 cases of local tenderness were significantly relieved. In this study, a fixed head rest or a body membrane was used to avoid and reduce patient displacement during the implantation process. The results of this study show that 125 I radioactive particle therapy is minimally invasive, safe, and can significantly improve the quality of life of patients in the short term.

Dosage determination is an important part of $125 \mathrm{I}$ seed implantation. Before TPS, the ideal dose distribution is automatically designed according to the tumor boundary, important tissue location, prescription dose, particle activity, etc., after the operation, the target area dose and the prescription dose are verified after the operation. Errors etc. evaluate the quality of radioactive seed implantation. In this study, according to the "Specifications for the Clinical Application of Radioactive Particles in Tumor Therapy", the prescribed dose of radiotherapy was set to 110-140 Gy [5]. If the dose is too small, the purpose of killing the tumor will not be achieved; if the dose is too large, it will Cause damage to normal tissues and organs around the lesion. In this study, D90, V100, HI and other dosimetry parameters were not statistically significant before and after surgery, and D90 reached (12 378.8 \pm 3 182.0) cGy and V100 reached $(92.2 \pm 14.3) \%$ after surgery, indicating that radioactive seed implantation The expected plan distribution is basically achieved, and the dosimetry requirements can be better met according to the TPS preoperative plan. However, the $\mathrm{HI}$ before and after the operation in this study was low, and the postoperative verification V150 was more than $50 \%$, suggesting that the dose distribution in the target area is uneven and the volume of the high-dose area is too large. Analyze the possible reasons for the error: (1) Postoperative changes in the size of the lesion and rapid reduction of edema around the lesion accelerate the dispersion and regression of radiation doseand reduce the conformation index of the dose distribution within the lesion [14-19]; and The verification dose is not the actual absorbed dose of the tumor. In this study, the postoperative verification time was within 24 hours. Local tissue edema that did not resolve within a short period of time after surgery would affect the assessment of the biological effect dose [18]; (2) due to postoperative CT Artifacts, imaging of a particle on two levels and overlapping of some particles make it difficult to identify the precise position of the particle, leading to a deviation in the postoperative verification and measurement dose; (3) The density of the tumor is different due to hemorrhage or necrosis due to particle radiation effects. This produces a change in particle position. In addition, postoperative verification found that 2 patients had progressed to the target lesion about 4 months after implantation treatment. Further research found that their postoperative D 90 was significantly lower than the prescribed dose planned before surgery. The main considerations are as 
follows: (1)2 The structure of the operation area was disordered, and the patient's age was too old, and the neck tissue was soft, which made implantation deviations during the operation; (2) Before and after the operation, the local muscles contracted due to posture examination, intraoperative anesthesia, and puncture stimulation. The shape of the volume changes.

RAIR-DTC lymph node metastasis is mostly close to the body surface, and the $y$-rays released by $125 \mathrm{I}$ particles can cause DNA double-strand breaks and affect skin cell metabolism [20]. Two patients in this study developed painful erythema, pigmentation after 1 week of anti-inflammatory treatment, and no skin ulceration. The analysis was due to multiple lesions being superficial and close to each other. With the elimination of tumor tissue in the coverage area, particles occurred Caused by the formation of local "hot spots" after aggregation. This suggests that when implanting particles, the distance between the particles and the skin surface should be at least $1 \mathrm{~cm}$, and the activity and number of particles should be appropriately reduced according to the anatomical part of the lesion.

In summary, $125 \mathrm{I}$ seed implantation for the treatment of RAIR-DTC lymph node metastasis has the advantages of good curative effect and fewer complications. The use of TPS can make the target dose conformity better, effectively kill the tumor and better protect the surrounding normal tissues. However, this study is a single-center retrospective study with a small sample size and a short follow-up period. It is necessary to expand the sample size and analyze the effects of factors such as dose and tumor size on the efficacy to provide better guidance for the standardized diagnosis and treatment of $125 \mathrm{I}$ particles.

\section{Declarations}

\section{Acknowledgements}

None.

\section{Authors'contributions}

ZGX carried out the studies,HSS and WGZ participated in collecting data, and drafted themanuscript. ZWW and JLQ performed the statistical analysis and participated in its design. GXY $\square L G$ and WYC helped to draft the manuscript. All authors read and approved the final manuscript.

\section{Funding}

Natural Science Foundation of Liaoning Province (20180551010)

\section{Availability of data and materials}

No additional data are available. 


\section{Ethics approval and consent to participate}

The study protocol was approved by the General hospital of northern theater command, and the participants provided written informed consent.

\section{Consent for publication}

Not applicable

\section{Competing interests}

All authors declared that there are no conflicts of interest.

\section{References}

1. Fahiminiya S, de Kock L, Foulkes WD. Biologic and clinical perspectives on thyroid cancer $\ \mathbf{J}$. N Engl J Med, 2016, 375(23): 2306-2307. DOI:10.1056/NEJMc1613118.

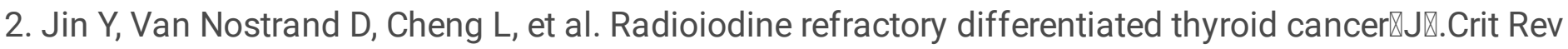
Oncol Hematol, 2018, 125: 111-120. DOI:10.1016/j.critrevonc.2018.03.012.

3. Chasseray M, Dissaux G, Bourbonne V, et al. Dose to the penile bulb and individual patient anatomy are predictive of erectile dysfunction in men treated with $125 \mathrm{I}$ low dose rate brachytherapy for localized prostate cancer囚J囚. Acta Oncol, 2019, 58(7): 10291035.DOI:10.1080/0284186X.2019.1574981.

4. Schmidt $A$, Iglesias $L$, Klain $M$, et al. Radioactive iodine-refractory differentiated thyroid cancer: an

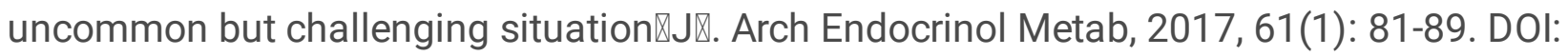
10.1590/2359-3997000000245.

5. Wang JJ. Standard for clinical application of radioactive particles in the treatment of tumor区M】. Beijing: Peking University Medical Press, 2011: 24.

6. Shiraishi Y, Yorozu A, Ohashi T, et al. A dose-response analysis of biochemical control outcomes after

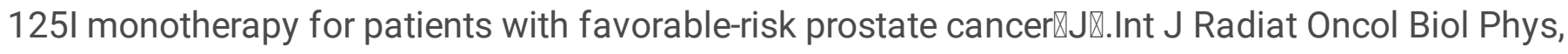
2014, 90(5): 1069-1075. DOI:10.1016/j.jirobp.2014.08.340.

7. Eisenhauer EA, Therasse P, Bogaerts $\mathrm{J}$, et al. New response evaluation criteria in solid tumours: revised RECIST guideline (version 1.1)囚J囚. Eur J Cancer, 2009, 45(2): 228-247.

DOI:10.1016/j.ejca.2008.10.026.

8. Bradley JD, Hope A, El Naqa I, et al. A nomogram to predict radiation pneumonitis, derived from a

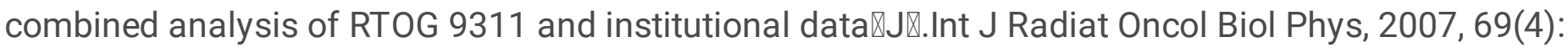
985-992. DOI:10.1016/j.jijrobp.2007.04.077. 
9. Zheng W. Changes in PD-1/PD-L1 pathway function in non-small-cell lung cancer tissue before and after $125 \mathrm{I}$ seed implantation $₫ \mathrm{~J} \rrbracket$. J Hainan Med Univ, 2017, 23(13): 1829-1832.

DOI:10.13210/j.cnki.jhmu.20170809.028.

10. Zhang J, Zhu Y, Dong M, et al. lodine-125 interstitial brachytherapy reduces tumor growth via

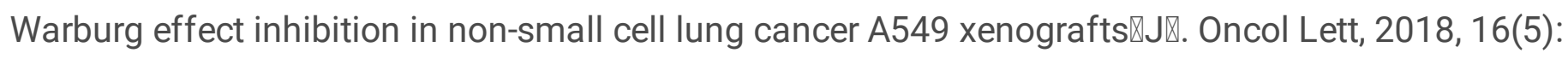
5969-5977. DOI:10.3892/ol.2018.9346.

11. Zhu Y, Dong M, Yang J, et al. Evaluation of iodine-125 interstitial brachytherapy using micro-positron emission tomography/computed tomography with $18 \mathrm{~F}$-fluorodeoxyglucose in hepatocellular

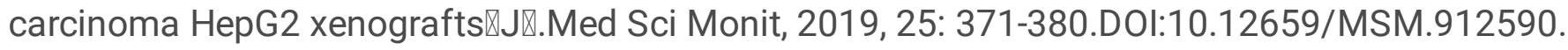

12. Chen W, Xie F, Zhang MB, et al. Ultrasound guided 125I brachytherapy in iodine refractory

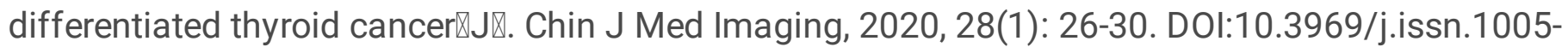
5185.2020.01.006.

13. Zhang WW, Hao SH, Wang ZG, et al. Clinical value of $125 \mathrm{I}$ seeds implantation in treatment of lymph nodes metastases from $131 \mathrm{I}$ refractory differentiated thyroid carcinoma $\mathbb{\mathrm { J }} \mathrm{J}$. Chin J Nucl Med Mol Imaging, 2018, 38(1): 9-13. DOI:10.3760/cma.j.issn.2095-2848.2018.01.003.

14. Xing C, Zhang KX, Yuan QQ, et al. Dosimetry verification of radioactive seed implantation for malignant tumor assisted by 3D-printing coplanar template囚J囚. Chin J Radiol Med Prot, 2017, 37(7): 514-517. DOI:10.3760/cma.j.issn.0254-5098.2017.07.008.

15. Du P, Xiao Y, Lu W. Modified fan-shaped distribution technology for computed tomography (CT)guided radioactive seed implantation in lung cancer patients with lung dysfunction $\mathbb{J}$ \. Med Sci Monit, 2017, 23: 4366-4375. DOI:10.12659/msm.902105.

16. Mukai Y, Hayashi N, Koike I, et al. Acute and late toxicities in localized prostate cancer patients treated with low-dose $125 \mathrm{I}$ brachytherapy $(110 \mathrm{~Gy})$ in combination with external beam radiation therapy versus brachytherapy alone (160 Gy) खJ区. J Contemp Brachytherapy, 2018, 10(5): 397-404. DOI:10.5114/jcb.2018.79379.

17. Wu J, Wang J, Sui AX, et al. Effect of tumor target volume reduction on dose after $125 \mathrm{I}$ seeds implantation囚J囚. Chin J Exp Surg, 2015, 32(2): 309. DOI:10.3760/cma.j.issn.1001-9030.2015.02.033.

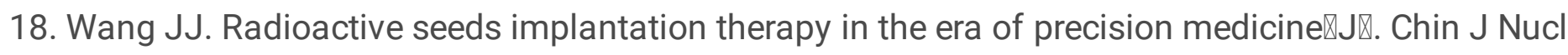
Med Mol Imaging, 2018, 38(1): 1-3. DOI:10.3760/cma.j.issn.2095-2848.2018.01.001.

19. Zhao XZ, Zhang HT, Di XM, et al. Relationship between the peripheral dose and radioactive counts of 125I seeds detected by SPECT/CT囚J囚. Chin J Nucl Med Mol Imaging, 2017, 37(6): 351-354. DOI:10.3760/cma.j.issn.2095-2848.2017.06.007.

20. Lin Q, Zhang Y, Dai JJ, et al. CT-guided 125 I seed brachytherapy for superficial lymph node

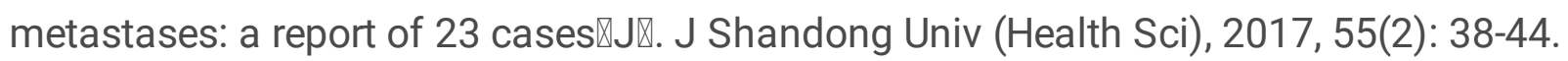
DOI:10.6040/j.issn.1671-7554.0.2016.1350.

\section{Figures}




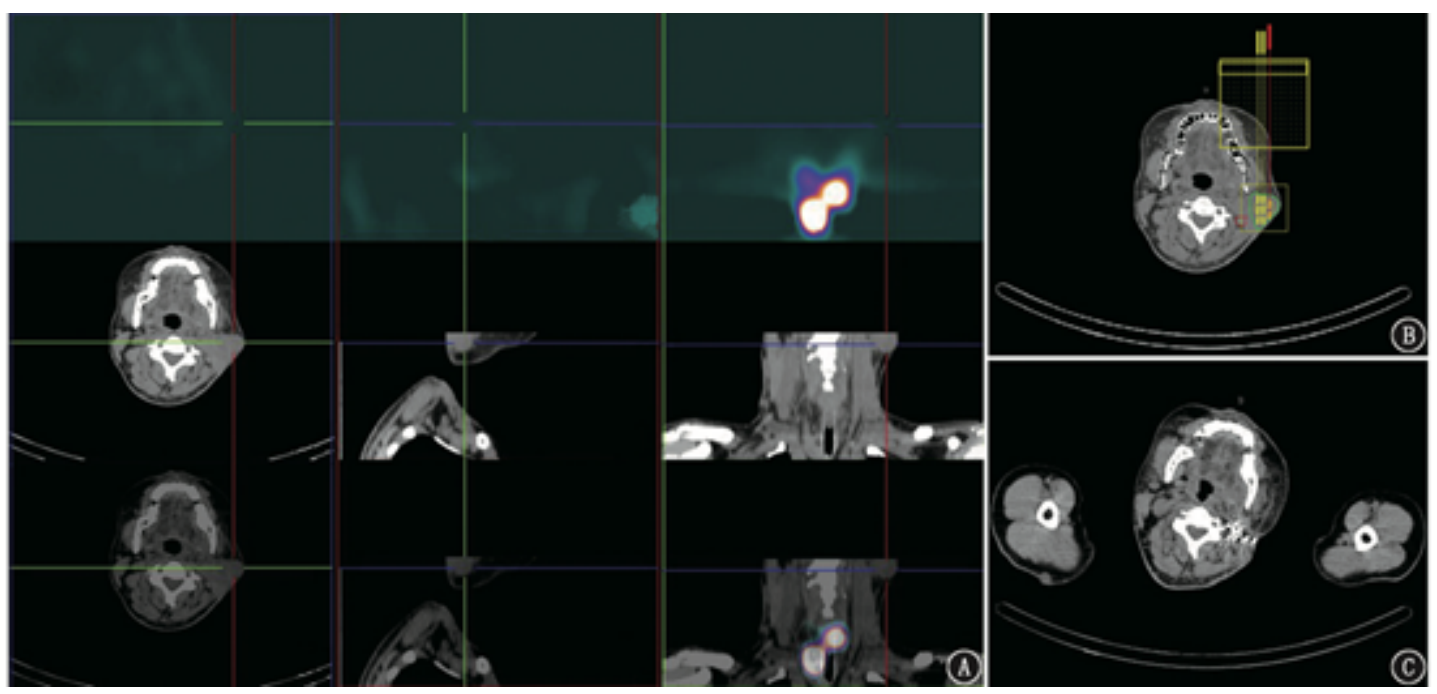

\section{Figure 1}

131I SPECT/CT imaging of a patient with 131I refractory differentiated thyroid cancer with metastasis to the left neck lymph node (male, 37 years old) before and after $125 \mathrm{I}$ seed implantation (the cross and the arrow show the lesion). A. 131I SPECT/CT neck fusion imaging showed no iodine uptake in the left cervical lymph node metastases; B. Treatment planning system (TPS) preoperative needle placement; C. Reexamination of the left cervical lymph node 6 months after treatment Significantly shrink, particle aggregation, local skin pigmentation 